$\begin{aligned} & 10 \begin{array}{l}\text { http://dx.doi.org/10.21611/qirt.2010.136 } \\ 10^{\text {th }} \text { International Conference on Quantitative InfraRed Thermography }\end{array} \\ & \text { July 27-30, 2010, Québec (Canada) }\end{aligned}$

\title{
Dual-Mode Photoacoustic Phased-Array Imager for Biomedical Applications
}

By Sergey Telenkov and Andreas Mandelis

Center for Advanced Diffusion-Wave Technologies (CADIFT), Dept. of Mechanical and Industrial

Engineering, University of Toronto, Toronto, Ontario, Canada M5S 3G8

sergeyt@mie.utoronto.ca

mandelis@mie.utoronto.ca

\section{Abstract}

Conventional pulse-echo ultrasound is routinely used in medical practice for diagnostic imaging. Despite its obvious success, traditional ultrasound has low-contrast when the targeted tissues lack echogenic features. Pulsed laser photoacoustics was developed as a hybrid technology taking advantage of high-contrast optical methods and high-resolution ultrasound. We present a novel photoacoustic imager that utilizes a conventional ultrasonic phasedarray sensor for rapid scanning of the targeted tissues and features dual-mode operation: traditional pulsed timedomain and frequency-domain photoacoustic method with chirped optical excitation (the photoacoustic radar or sonar). Typical characteristics of the imager and potential for biomedical applications will be discussed.

Ultrasonic imaging of extended tissue volumes requires continuous scanning of a transducer sensor with simultaneous data acquisition and processing. Use of a single element transducer for two-dimensional "slice" or three-dimensional volume imaging is very time consuming and cannot be used for real-time ultrasonic imaging especially when the targeted tissues, such as heart, are constantly moving. Clinical demand for real-time diagnostic imaging resulted in rapid advancement of acoustic sensor technology and proliferation of imaging systems utilizing multi-element sensor arrays. Advantage of arrays consists in their ability to sweep ultrasonic beam over the test volume without moving the array probe. Specific implementation of the scanning mechanism varies for linear and phased-arrays but either case involves electronic delay or phase shifting of the individual sensor signals for dynamic beam steering and focusing. In recent years, the ultrasonic transducer arrays were actively used in laser photoacoustics for variety of applications ranging from a small animal imaging to visualisation of human vasculature and breast cancer detection. In the present work, we describe a novel photoacoustic imager utilizing a conventional phased-array ultrasonic probe and the dual-mode imaging system for biomedical applications. Schematic diagram of the imager sensor probe is shown in Figure 1a.

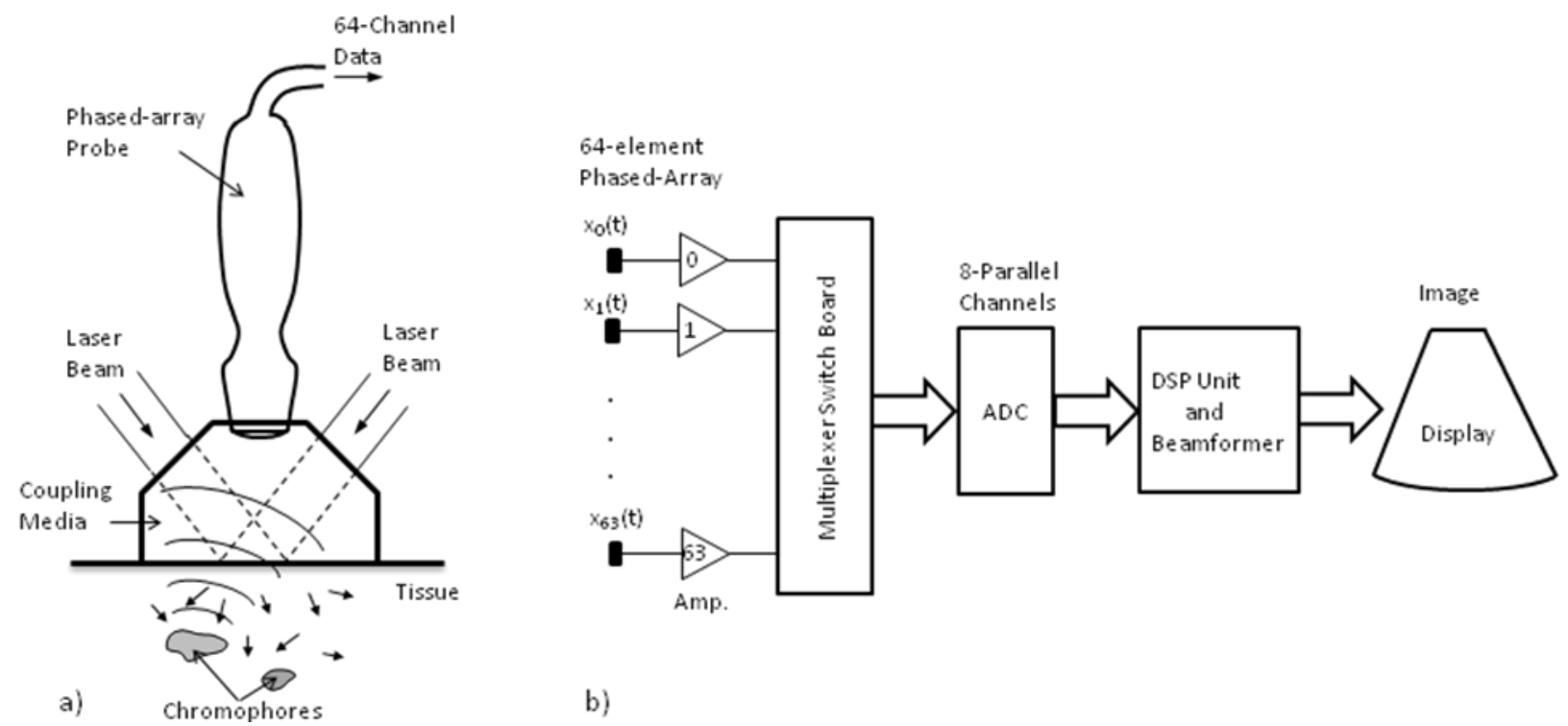

Figure 1. Schematics of the imager probe (a) and the data flow (b) in the 64-channel photoacoustic imager. 
The phased-array probe contains 64 transducer elements assembled with the pitch of $254 \mu \mathrm{m}$. The central frequency of each transducer element is $2.5 \mathrm{MHz}$ and the bandwidth $1.5 \mathrm{MHz}$. Data acquisition and signal processing was implemented using National Instruments (Texas, Austin) modular instruments and LabView programming environment, respectively. The diagram describing the imager key components and data flow is shown in Figure $1 \mathrm{~b}$. Readout of 64 data channels is realized using a multiplexer board with 8 parallel channels digitized simultaneously at the $60 \mathrm{MHz}$ sampling rate. Image reconstruction similar to the conventional ultrasonic B-scan is accomplished using all 64 digitized signals and a beamforming algorithm for dynamic beam steering and focusing. Unique feature of the present imaging system is ability to operate in two modes: traditional pulsed time-domain with nanosecond laser irradiation and the frequency-domain photoacoustic imaging with chirped optical excitation. The latter mode is similar to radar operation therefore it can be called a photoacoustic radar or sonar. Use of the frequency-modulated waveforms and signal compression was reported in earlier studies of both conventional pulse-echo ultrasound and photoacoustic imaging. It was found that the coded excitation can be used to improve the signal-to-noise ratio and overall image contrast. We analyse both photoacoustic imaging modes using tissue phantoms and in-vivo tissue specimens. 